\title{
A combined CFD-experimental method for abrasive erosion testing of concrete
}

\author{
Gianandrea Vittorio Messa ${ }^{1 *}$, Renan De Lima Branco ${ }^{2}$, José Gilberto Dalfré Filho ${ }^{2}$, Stefano Malavasi ${ }^{1}$ \\ ${ }^{1}$ FLUIDLab group, Department of Civil and Environmental Engineering, Politecnico di Milano, Piazza Leonardo Da Vinci, 32, 20133 \\ Milano, Italy. \\ ${ }^{2}$ College of Civil Engineering, Architecture and Urbanism, University of Campinas, Av. Albert Einstein, 951, Campinas, SP, Brazil. \\ ${ }^{*}$ Corresponding author. Tel.: +39 022399 6287. E-mail: gianandreavittorio.messa@polimi.it
}

\begin{abstract}
Serious damage may occur to concrete hydraulic structures, such as water galleries, spillways, and stilling basins, due to the abrasive erosion caused by the presence of solid particles in the flow. This underlines the importance of being capable in providing characterization of the concrete from the point of view of its vulnerability to abrasive erosion, in order to improve the design of the structure and the material selection. Nevertheless, the existing apparatus for concrete abrasive erosion testing are either far from allowing realistic simulation of the actual environment in which this phenomenon occurs, or show a large degree of complexity and cost. An alternative method has been developed with the aid of Computational Fluid Dynamics (CFD). CFD was first employed to verify the effectiveness of a new laboratory equipment. Afterwards, a parameter has been introduced which, by successful comparison against preliminary experiments, proved suitable to quantify the effect of the fluid dynamic conditions on the concrete abrasive erosion, thereby opening the way to CFD-based customization of the apparatus. In the future, the synergy of numerical and physical modelling will allow developing predictive models for concrete erosion, making it possible to reliably simulate real structures.
\end{abstract}

Keywords: Abrasive erosion; Computational fluid dynamics; Concrete; Experiments; Two-phase flow.

\section{INTRODUCTION}

The abrasive erosion of hydraulic concrete structures is the progressive disintegration of the material caused by watertransported solid, sand, gravel, ice, or debris (Graham, 1998). The irreversible nature of this damage, and the high costs related to this effect, makes the capability of correctly characterizing the concretes from the point of view of their resistance to abrasive erosion very important.

According to Liu et al. (2006), the mass loss caused by abrasive erosion in hydraulic concrete is a three-stage process. Initially, the pressure of water molecules causes a pre-abrasion peeling of the concrete surface. Afterwards, the impacts of the solids carried along with the water result in the removal of the mortar and the subsequent exposure of the coarse aggregates. Finally, the removal of the aggregates occurs due to scratching and shearing actions.

A number of test methods have been proposed by the ASTM committee to test the abrasion resistance of concrete. One out of them, referred to as "underwater method", is specifically intended to simulate the abrasive action of waterborne particle under water (ASTM C1138M, 2012). The apparatus, sketched in Fig. 1a, consists of a steel cylindrical tank filled with water at the bottom of which a concrete specimen is placed. An agitator with a specially designed paddle keeps the water and the abradant in rotary motion. The abradant consists of 70 steel grinding balls with nominal size ranging between 12.6 and $25.3 \mathrm{~mm}$. This equipment was used by several authors to investigate the relative resistance of different concretes under an erosive action (e.g. Dalfré Filho et al., 2000; Horszczaruk, 2005, 2008, 2009; Kumar and Sharma, 2014; Mohebi et al., 2015; Yen et al., 2007). However, despite the attractive simplicity of the ASTM C1138M apparatus, its main problem is that it involves different erosion mechanisms than those encountered in practical applications.
Liu et al (2006, 2012) and Dandapat and Deb (2016) used a different setup, in which a concrete specimen is exposed, at a given inclination angle, to a slurry sand-water jet coming out from a nozzle (Fig. 1b). The large, rectangular nozzle and the inclination angle were defined in such a way to reproduce the water flow over a spillway. Moreover, the mass loss is due to normal impacts and shearing actions, further approaching the actual conditions. Nevertheless, the dimensions of the rig are considerable, having technical and economic implications.

The apparatus shown in Fig. 1c was developed by Horszczaruk $(2000,2004)$. It consists of a steel drum, partially filled with a mixture of aggregates and water. A rotating shaft with several concrete samples allows the interaction between concrete and abrasive mixture. The goal of better simulating the natural conditions in which concrete abrasion occurs was achieved, but, at the same time, the size of the rig and the difficulties in controlling the experimental conditions for such complex flow appear the key drawbacks of this solution.

The above discussion indicates that none of the available methods for concrete erosion testing is free from critical aspects, mainly residing in either difference from the actual working conditions (for the ASTM "underwater method") or excessive complexity of the setup (for the abrasive jet method and the rotating device). In the present work, an alternative testing strategy is proposed. This is not limited to the development of a new apparatus, designed with the aid of Computational Fluid Dynamics (CFD), which combines the earlier ones integrating the advantages from each of them. Actually, it involves a strong synergy between numerical and physical modelling, a concept which has already been explored in erosion studies (e.g. Clark, 2002; Gnanavelu et al., 2011; Mansouri et al., 2015). A parameter has been introduced which, at the present stage, represents only the effect of the fluid dynamic conditions on the effectiveness of the apparatus. This allows customization of the setup to optimize its performance for specific testing conditions. 
(a)

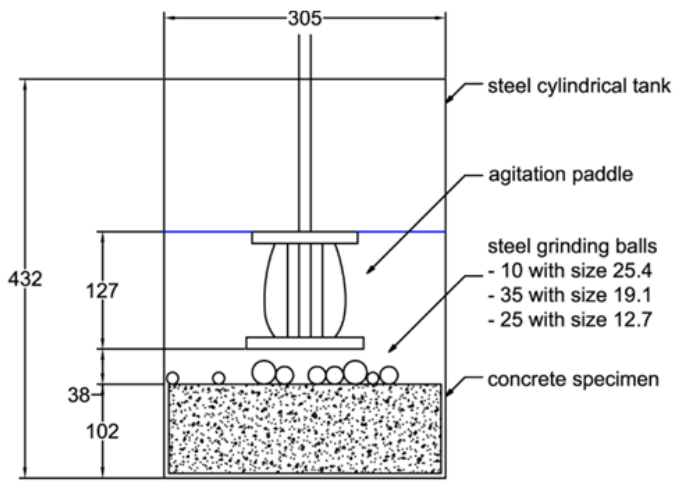

(b)
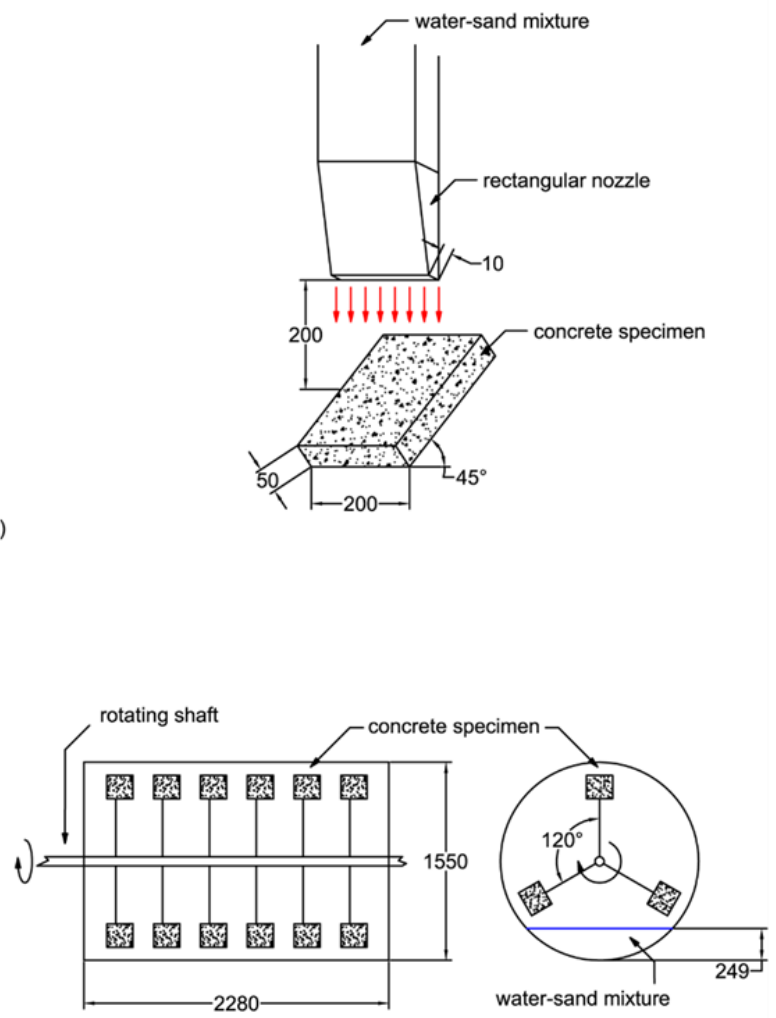

(c)

Fig. 1. Main details of three existing setups for underwater erosion testing: (a) the ASTM C1138M (2012) apparatus; (b) nozzlespecimen system used by Liu et al. $(2006,2012)$; (c) the device proposed by Horszczaruk $(2000,2004)$. Dimensions are in $\mathrm{mm}$.

Laboratory experiments will be then performed on different concretes and, based on the acquired data, the proposed parameter will be made material-dependent and turned into a predictive erosion model. This will open the way to the reliable simulation of hydraulic structures, such as water galleries, spillways, and stilling basins. In addition, the laboratory apparatus may be also used as an alternative to the ASTM C1138M for comparing the resistance of various concrete formulations.

The remainder of the paper is divided in three sections, followed by the conclusions. In the first one, the proposed setup is described. In the second one, the CFD model used for simulating the liquid-solid flow in the system is illustrated, together with the followed computational methodology. In the third one, the effectiveness of the equipment is demonstrated, also by comparison to the outcomes of some preliminary tests performed in a prototype apparatus.

\section{THE PROPOSED APPARATUS}

The new device is a modification of the ASTM C1138M method in which the steel balls have been replaced by the smaller granular material encountered in actual environments. Moreover, the agitator has been changed with a Pitched Blade design and four lateral baffles have been introduced (Fig. 2), in order to create a downward flow with enhanced turbulence, which promotes the impingements of the particles against the concrete base. These modifications concur to make the testing conditions closer to those encountered by concrete hydraulic structures, in terms of involved materials and erosion mechanisms, without any increase in technical and economic burden.
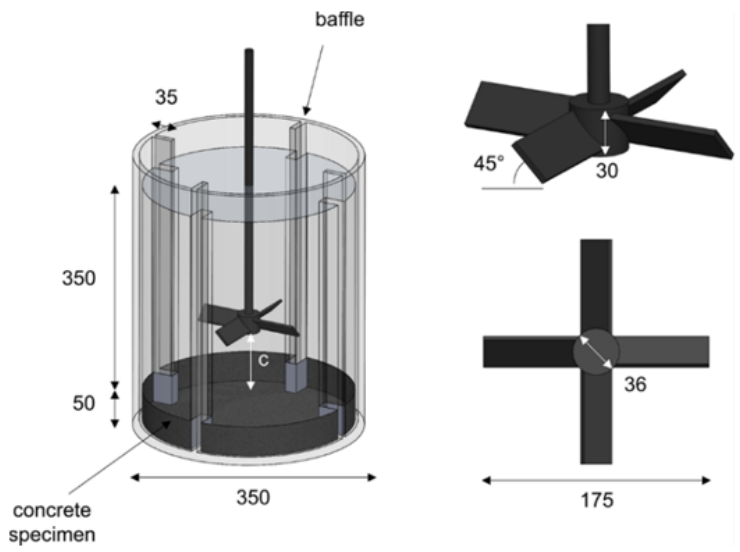

Fig. 2. The apparatus proposed in this study. Dimensions are in $\mathrm{mm}$.

The dimensions reported in Fig. 2 and their ratios were defined based on analogy with the ASTM C1138M and the stirred tank used by Fokema et al. (1994). The tank diameter, $T$, is 350 $\mathrm{mm}$, and it is equal to the still water depth above the specimen, which is located at the bottom and has a height equal to $50 \mathrm{~mm}$. The width of the four, equally spaced, lateral baffles is $35 \mathrm{~mm}$ $(T / 10)$. The Pitched Blade impeller has angle of $45^{\circ}$, and diameter equal to $175 \mathrm{~mm}(T / 2)$. As it will be clarified later, the rotational velocity of the impeller, $\Omega$, and its clearance, $c$ (that is, its distance from the concrete specimen) have significant influence on the development of the erosion phenomenon, and, therefore, they were the subject of specific analyses. In this paper, the abradant consists of $950 \mathrm{~g}$ of silica sand particles with density equal to $2650 \mathrm{~kg} / \mathrm{m}^{3}$, yielding a static solid volume fraction of $1 \%$. In the laboratory experiments, the particle size ranges within 1.2 and $2.0 \mathrm{~mm}$ (the average being $1.5 \mathrm{~mm}$ ), as determined by a sieve classification performed before the test. In the numerical simulations, the solid phase is modelled as monodispersed with size equal to $1.5 \mathrm{~mm}$. The carrier fluid is water at $20^{\circ} \mathrm{C}$ with density and kinematic viscosity coefficient equal to $998.23 \mathrm{~kg} / \mathrm{m}^{3}$ and $1 \mathrm{e}-6 \mathrm{~m}^{2} / \mathrm{s}$, respectively.

\section{METHODOLOGY \\ Governing equations}

Even if the average abrasive content in the tank is low, the relatively high solid volume fraction occurring in certain regions makes it necessary to account for two- and four-way coupled interactions between the phases. Therefore, the liquidparticle flow was modelled based on the Euler-Euler approach, in which both phases are interpreted as interpenetrating continua. Particularly, use is made of the extension to dense flow of the IPSA model of Spalding (1980) which has been proposed by Messa and co-workers (2013, 2014, 2014a, 2014b, 2015). 
The flow is assumed statistically steady and so the mass and momentum conservation equations for each phase are, respectively

$$
\begin{aligned}
& \nabla \cdot\left(\alpha_{q} \rho_{q} \mathbf{U}_{q}\right)=\nabla \cdot\left(\rho_{q} \frac{v_{t}}{\sigma_{\alpha}} \nabla \alpha_{q}\right) \\
& \nabla \cdot\left(\alpha_{q} \rho_{q} \mathbf{U}_{q} \mathbf{U}_{q}\right)-\nabla \cdot\left(\alpha_{q} \rho_{q} v_{q} \nabla \mathbf{U}_{q}\right)-\nabla \cdot\left(\alpha_{q} \rho_{q} v_{t} \nabla \mathbf{U}_{q}\right) \\
& -\nabla \cdot\left(\mathbf{U}_{q} \rho_{q} \frac{v_{t}}{\sigma_{\alpha}} \nabla \alpha_{q}\right)=-\alpha_{q} \nabla P+\alpha_{q} \rho_{q} \mathbf{g}+\mathbf{M}_{q}
\end{aligned}
$$

where the subscript $q$ is a phase indicator parameter equal to $f$ and $p$ for the fluid and the particles, respectively. Moreover, $\alpha_{q}$ is the local average volume fraction, $\rho_{q}$ is the density, $\mathbf{U}_{k}$ is the average velocity vector, $\sigma_{\alpha}$ is the turbulent Schmidt number for volume fractions, set equal to $0.7, v_{q}$ is the kinematic viscosity coefficient, $P$ is the average pressure, $v_{t}$ is the eddy viscosity, and $\mathbf{g}$ is the gravitational acceleration vector.

The viscosity of the solids is obtained from a friction, mixture-viscosity related parameter, $\mu_{m}$, by assuming the following linear relationship:

$\mu_{m}=\alpha_{f} \rho_{f} v_{f}+\alpha_{p} \rho_{p} v_{p}$

and evaluating $\mu_{m}$ by means of the comprehensive formula proposed by Cheng and Law (2003):

$\mu_{m}=\rho_{f} v_{f} \exp \left\{\frac{2.5}{\beta}\left[\frac{1}{\left(1-\alpha_{p}\right)^{\beta}-1}\right]\right\}$

in which $\beta$ is a material-dependent coefficient that, as suggested, was set to 3 in this paper.

The interfacial momentum transfer term $\mathbf{M}_{q=f, p}$ is given by:

$$
\mathbf{M}_{f}=-\mathbf{M}_{p}=\frac{3}{4 d_{p}} C_{d} \alpha_{p} \rho_{f}\left|\mathbf{U}_{p}-\mathbf{U}_{f}\right|\left(\mathbf{U}_{p}-\mathbf{U}_{f}\right)
$$

where $d_{p}$ is the particle size, and $C_{d}$ is the drag coefficient, which is related to the $\mu_{m}$-based particle Reynolds number, $\operatorname{Re}_{p}=\rho_{f}\left|\mathbf{U}_{p}-\mathbf{U}_{f}\right| d_{p} / \mu_{m}$, via the correlation of Shiller and Naumann (1935):

$C_{d}=\max \left[\frac{24}{\operatorname{Re}_{p}}\left(1+0.15 \operatorname{Re}_{p}^{0.687}\right), 0.44\right]$

The eddy viscosity, $v_{t}$, was evaluated by an extension of the standard high-Reynolds form of the k- $\varepsilon$ model of Launder and Spalding (1974) to two-phase flows, which is well documented in Messa et al. (2013, 2014, 2015).

\section{Computational domain and boundary conditions}

The computational domain is shown in Fig. 3, where it is evident that the geometrical symmetry of the system has been exploited by solving only over a quarter of the tank.

The boundary conditions are zero shear stress, cyclic, and solid walls. Particularly, zero shear stress has been imposed to both phases in correspondence to the upper boundary, in order to reproduce the free surface of the mixture, assumed flat. The two vertical, planar surfaces are attributed a cyclic condition to account for the effect of the remaining three quarters of the tank.

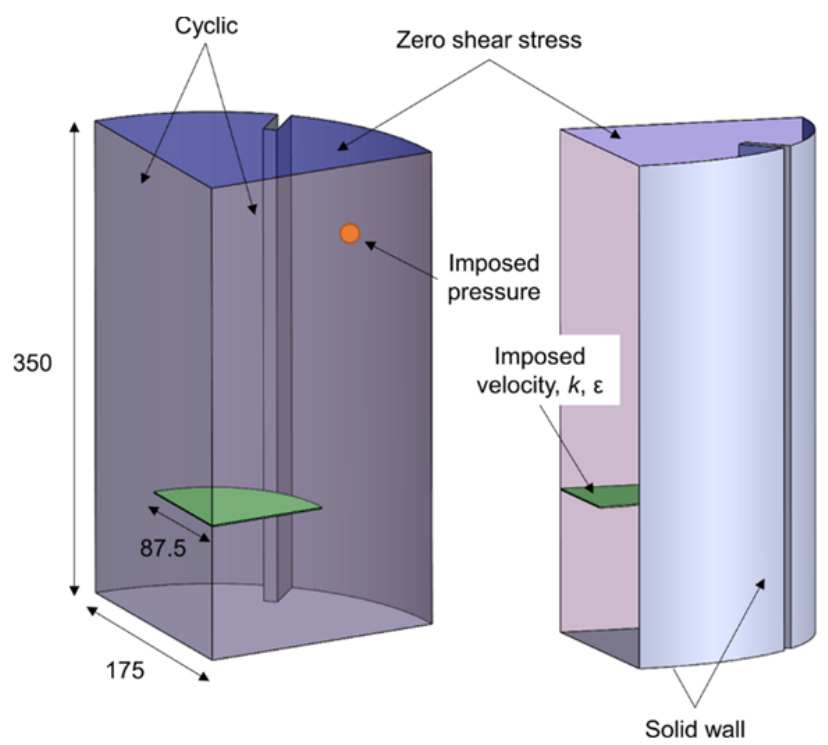

Fig. 3. Sketch of the computational domain and the imposed boundary conditions.

Finally, all other boundaries (i.e. the specimen, the tank wall, and the baffles) are modelled as solid walls. There, the liquid has zero velocity; the wall shear stress of this phase, and the turbulent kinetic energy and the dissipation rate in the near-wall cells are obtained from the equilibrium wall function of Launder and Spalding (1972) for smooth walls. Conversely, the zero wall shear stress condition has been applied to the solid phase.

In order to make the pressure distribution uniquely determined, the pressure was fixed in a cell of the computational domain. The average volume fraction in the tank was imposed as initialization step of the solution algorithm. Finally, the effect of the rotating impeller on the mean flow was modelled by imposing the velocity of both phases and the turbulent parameters on a surface with the shape of a quarter of a disk. Further details on this aspect are provided in Appendix A.

\section{Computational methodology and consistency of the numerical solution}

The commercial CFD code PHOENICS 2014 was employed for the numerical solution of the Euler-Euler model equations. Particularly, use was made of a recompiled version of the solver together with user-defined subroutines. The equations have been discretized using the finite volume method, and the calculations performed following the elliptic-staggered formulation, in which the scalar variables are evaluated at the cell centers and the velocity components at the cell faces. Central differencing is employed for the diffusion terms, while the convection terms are discretized using the hybrid differencing scheme of Spalding (1972). The finite-volume equations are solved by means of the IPSA algorithm of Spalding (1980). The calculation procedure is organized in a slab-by-slab manner, in which all the dependent variables are solved at the current slab before the solver routine moves to the next slab. The numerical solution procedure requires appropriate relaxation of the field variables to achieve convergence.

A cylindrical-polar structured mesh was used to discretize the domain. With the Euler-Euler model, the definition of the mesh is more delicate than in single-phase flow simulations, where the requirements are just grid-independence and consistency with the wall treatment approach. The averaging process at the basis of the flow equations implies that the cell size should be larger than the particle size in order to be physically 
consistent. Trying to generalize the conclusions of Picardi et al. (2016) to a 3D domain, we adopted the criterion that $\Delta r$ and $\Delta z$ should be at least equal to $\sqrt{2} d_{p}$, whilst $\Delta \theta$ was defined in such a way to produce an average cell-area in the $\theta-r$ plane not lower than $2 d_{p}^{2}$. The mesh below the impeller was the finest possible in compliance with the above constrains for $d_{p}=1.5 \mathrm{~mm}$, namely $\Delta \theta=0.0231 \mathrm{rad}, \Delta r=\Delta z=2.12 \mathrm{~mm}$. In order to reduce the computational burden of the simulations, the cells above the impeller were axially elongated in accordance to a 1.2 power law distribution, reaching a value of $\Delta z$ equal to about $6 \mathrm{~mm}$ close to the upper surface. Specific tests were carried out to demonstrate that such a grid coarsening did not affect the erosion-related parameters introduced in the next section.

The PHOENICS solver was run until the sum of the absolute residual over the whole solution domain is less than $0.01 \%$ of reference quantities based on the total inflow of the variable in question. This is typically achieved in about 12000 iterations.

\section{RESULTS}

\section{Effectiveness of the new apparatus}

In order to produce wear mechanisms that are similar to those encountered in concrete hydraulic structures (Graham, 1998), the new apparatus should be able to create regions in which the sand particles impinge against the bottom. Numerical simulations allowed verifying that the Pitched blade impeller fits into this purpose, being capable in pushing the particles against the specimen and, afterwards, re-lifting them up close to the tank wall. In fact, the mean velocity field of the solid phase, depicted in Fig. 4, clearly shows the presence of an annular zone where particle impingements are likely to occur. In Fig. 4, the impeller clearance is $c=5.6 \mathrm{~cm}$ (equal to 0.16 tank diameters, $T$ ) and its rotational speed is $600 \mathrm{rpm}$, but qualitatively similar solutions are obtained in all the simulations.

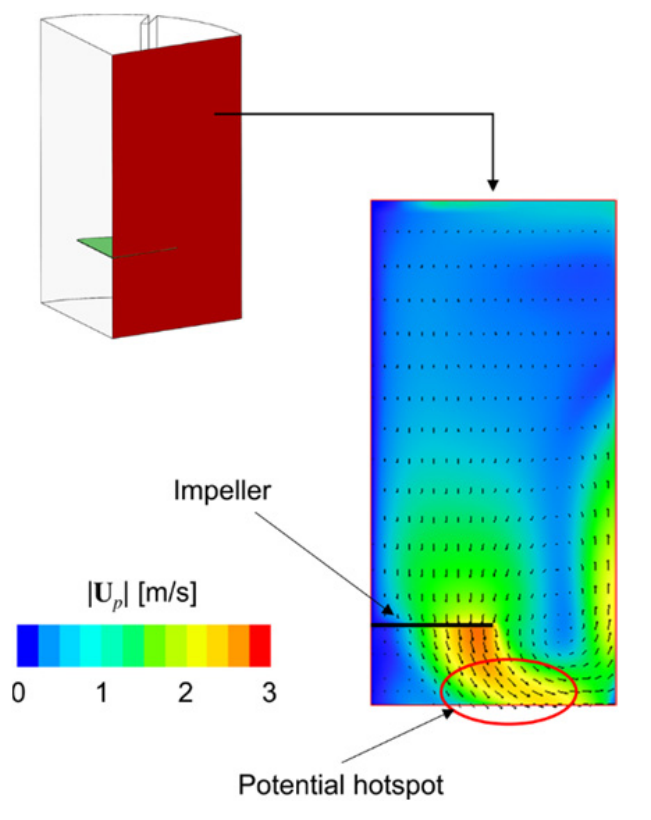

Fig. 4. Mean velocity field of the solid phase for a typical simulation.

\section{Definition of a new erosion-related function}

Usually, numerical investigations concerning solid particle erosion involve dilute flows, and they predict the loss of material by simulating the fluid-particle flow using an EulerianLagrangian two-phase model, in which a Lagrangian equation of motion is solved for a number of computational particles, followed by the application of a single-particle erosion model to each particle-wall impingement (Parsi et al., 2014).

Nevertheless, this approach is precluded in this study for a couple of reasons. First, it is not clear how to couple the EulerEuler fluid-dynamic solution with a single-particle erosion model. Second, it would be hard to guarantee the reliability of the wear estimates since they are strongly affected by the erosion model (Messa and Malavasi, 2017), which includes material-dependent constants obtained by fitting experimental data.

It is worth remarking that the numerical approach is here aimed at attaining rough estimation of how the fluid dynamic of the system affects the effectiveness of the apparatus, in order to create a tool that can be used in future experiment's design. Based on this consideration, we introduced a function, $\phi$, which can be regarded as an indicator of erosion in an Euler-Euler framework. This parameter may be used for comparing different operating conditions of the setup (e.g. in terms of type of impeller, clearance, and rotational speed), but it does not allow any quantitative prediction of mass loss, as it does not account for the characteristics of the target material. As already mentioned, turning $\phi$ into an erosion prediction model will be demanded to a later stage of this research project. The function $\phi$ is defined on a discretized plane parallel to the surface of the specimen (Fig. 5), as follows

$$
\phi=-\min \left(\dot{m}_{a d v, z}, 0\right)\left|\mathbf{U}_{p}\right|^{2} \frac{2 \theta_{p}}{\pi}
$$

where $\theta_{p}$ is the inclination angle of the mean solid phase velocity vector, and $\dot{m}_{a d v, \mathrm{z}}$ is the advective flux per unit area of the solid phase along direction $z$. All quantities above are interpolated at the centroid of each element of the plane, and the last two variables are calculated as

$$
\begin{aligned}
& \theta_{p}=\arctan \frac{W_{p}}{\sqrt{U_{p}^{2}+V_{p}^{2}}} \\
& \dot{m}_{a d v, z}=\rho_{p} \alpha_{p} W_{p}
\end{aligned}
$$

where $U_{p}, V_{p}$, and $W_{p}$ are the components of the mean solid phase velocity along directions $\theta, r$, and $z$, respectively.

The values of $\phi$ are different from zero only in those elements where the advective solid mass flux is directed downwards, thereby assuming that the others do not contribute to the erosion process. Moreover, the function is directly proportional to the flux of kinetic energy, and linearly increases with the inclination angle, $\theta_{p}$. By analogy with single-particle erosion models, $\phi$ was regarded as an indicator of the penetration rate, (i.e., the speed at which the scar depth increases) and, therefore, the integral of $\phi$ over the surface, referred to as $\Phi$, relates to the erosion rate, i.e. the velocity at which mass loss occurs. The monotonically increasing trend of erosion versus the inclination angle is typical of brittle materials (Parsi et al., 2014) and, in the absence of specific data for concrete other than the experiments of Liu et al. (2012), as a first step a linear approximation was assumed.

An issue arises from the fact that $\phi$ is zero over the surface of the specimen, since $\dot{m}_{a d v, \mathrm{z}}$ is zero there. Therefore, this function has been evaluated at a certain distance from the lower boundary, referred to as $\delta$ in Fig. 5. This approach introduces an arbitrary parameter in the computational model but specific sensitivity analyses showed that, generally, the relative effect of 


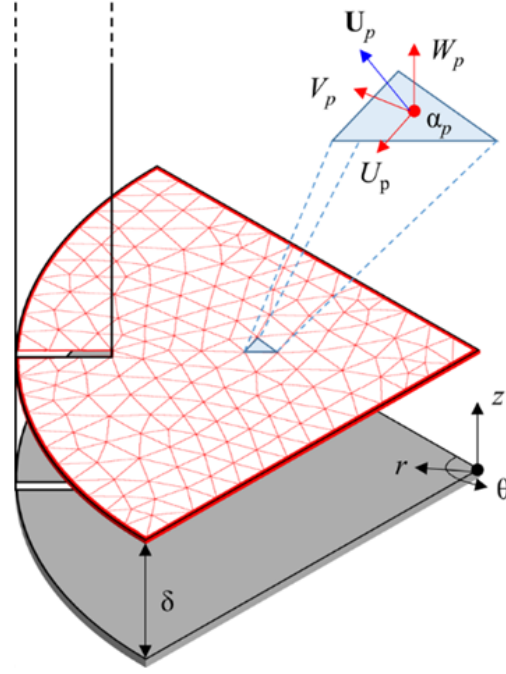

Fig. 5. Reference plane for evaluation of $\phi$.

the fluid dynamics of the system on $\phi$ is only moderately affected by $\delta$ when this parameter varies between 10 and $30 \mathrm{~mm}$. The results shown hereafter refer to $\delta=20 \mathrm{~mm}$.

A typical $\phi$ distribution is depicted in Fig. 6 for $c / T=0.16$ and $\Omega=600 \mathrm{rpm}$, showing consistency with the solid phase velocity vectors of Fig. 4 . The similarity between the ring shape of the function and that of the scar on the top of the specimen will be made evident in the next section, giving strength to the assumption that $\phi$ is related to the penetration rate.

A sensitivity analysis was performed to investigate the effect of the impeller clearance and the impeller rotational speed on the expected development of the erosion process. The ratio between the impeller clearance and the tank diameter, $c / T$, was varied in the range $0.16 \div 0.29$, and $\Omega$ increased from 500 to $700 \mathrm{rpm}$. Researches by Zwietering (1958), Mak (1992), and Jirout and Rieger (2011) suggest that, in all cases, the rotational speed is enough to avoid particle accumulation. The effects of the above parameters on the predicted wear scar are shown in Figs. 7(a) and (b), making reference to the profile of $\phi$ along the line midway between two baffles. The simulation suggest that $c / T$ and $\phi$ differently affect the erosion shape due to their effect on the fluid dynamics of the two-phase system. Particularly, the lifting the impeller upwards causes a decrease in the penetration rate accompanied by an increase in the inner radius of the scar ring. Conversely, a variation in the impeller speed results in a change in the velocity at which erosion occurs, but it is unlikely to produce a change in the shape of the scar.

The considerable influence of $c / T$ and $\phi$ on the erosion of the specimen is confirmed by the values of the integral of $\phi$, reported in Table 1 for all flow conditions.

Table 1. Values of $\Phi$ for different flow conditions.

\begin{tabular}{ccc}
\hline$c / T[-]$ & $\Omega[\mathrm{rpm}]$ & $\Phi\left[\mathrm{kg} \cdot \mathrm{m}^{2} / \mathrm{s}^{3}\right]$ \\
\hline 0.16 & 600 & 2.72 \\
0.22 & 600 & 1.90 \\
0.29 & 600 & 0.91 \\
0.16 & 500 & 1.75 \\
0.16 & 700 & 4.08 \\
\hline
\end{tabular}

\section{Comparison against experiments}

Prototypes of the apparatus were set up in accordance with the dimensions reported in Fig. 2, and the first experimental tests were performed in the Laboratory of Hydraulics and Fluid
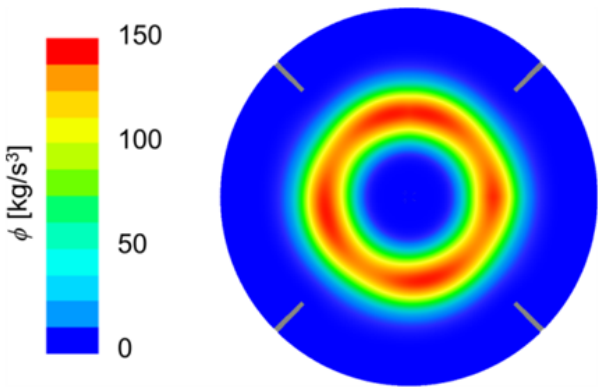

Fig. 6. Distribution of $\phi$ for $c / T=0.16$ and $\Omega=600 \mathrm{rpm}$.
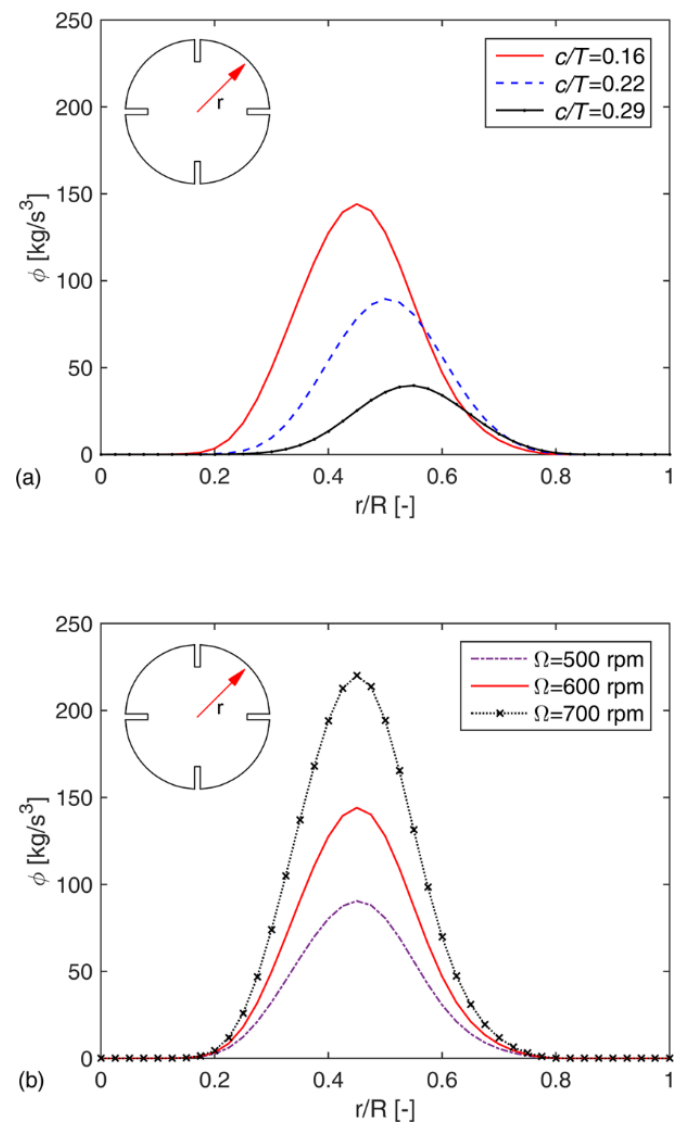

Fig. 7. Radial profile of $\phi$ along the line midway between two baffles: (a) effect of $c / T$ for $\Omega=600 \mathrm{rpm}$; (b) effect of $\Omega$ for $c / T=$ 0.1 .

Mechanics of the University of Campinas (Fig. 8). The tank and the baffles are made of acrylic Plexiglass to allow visual inspection of the test. The thickness of the tank wall is $5 \mathrm{~mm}$. The Pitched Blade impeller is kept into rotation by a 4-pole motor with nominal power of $370 \mathrm{~W}$, driven by a frequency inverter that allows varying the rotating speed up to $1800 \mathrm{rpm}$.

The specimens for the erosion tests were cylinders, which have been accurately re-shaped to fit into the baffled tank. The Brazilian Standard NBR 5738 (2003) was followed for their preparation. For the resistance compressive strength and elasticity modulus at 28 days, cylindrical samples of $10 \mathrm{~mm}$ diameter and $200 \mathrm{~mm}$ height were molded. It is again underlined that the objective of these preliminary experiments is the verification of the effectiveness of the apparatus and the above simulation methodology, whilst the use of the setup for characterizing con- 


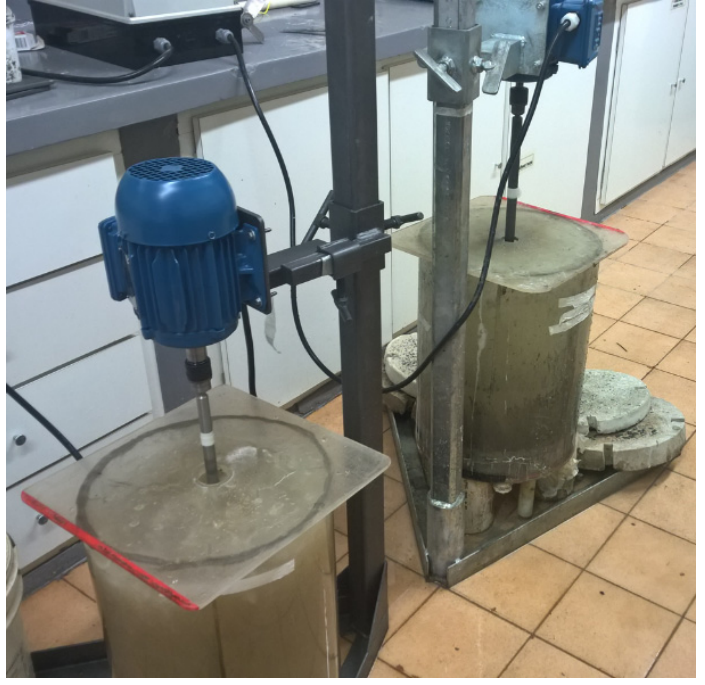

Fig. 8. The experimental setup at the University of Campinas.

cretes to be employed in practical applications is demanded to a later stage. Therefore, the mixture had an extremely high waterto-cement ratio, thus very low compressive resistance, to accelerate the erosion process and enhance the influence of the fluiddynamic parameters of the system. The samples have the cement-sand-rock composition of 1-6.47-5.29 and $w / c$ ratio of 1.47. As already mentioned, the carrier fluid is water, and the abradant consists of silica sand with size in the range $1.19 \div$ $2.00 \mathrm{~mm}$, the average being $1.5 \mathrm{~mm}$ (which is the value of $d_{p}$ considered in the numerical simulations).

The experimental methodology is as follows. Firstly, the concrete sample is weighted using a balance (Toledo, model PRIX III Fit, range $0.050-15.000 \mathrm{~kg}$, accuracy of $0.005 \mathrm{~kg}$ ) and its initial mass is recorded. Secondly, the sample is positioned at the bottom of the tank, and the gap between the specimen and the tank wall is filled with sealant material. Thirdly, clean water is poured into the tank up to a static level of $350 \mathrm{~mm}$ above the specimen. $0.950 \mathrm{~kg}$ of abradant was added to produce a static solid volume fraction equal to $1 \%$. Fourthly, after positioning the impeller-shaft motor assembly at the desired clearance, the motor is turned on and the rotational velocity is adjusted. Every 6 hours, the mass of the specimen was measured and the relative mass decrement, $\Delta M / M_{0}$, calculated. Each time, the abrasive mixture was renewed, in order to reduce as much as possible not only the self-enhancements of the erosion process due to the contribution of detached concrete pieces, but also the spurious effects due to particle degradation.

The experiments were made for two different impeller clearances, namely $c=56 \mathrm{~mm}(c / T=0.16)$ and $c=100 \mathrm{~mm}(c / T=$ $0.29)$, at constant impeller speed of $600 \mathrm{rpm}$. The overall testing time was 96 hours for the high clearance case, whilst it was reduced to 24 hours for the low clearance one due to the higher velocity at which erosion occurred.

The verification of the effectiveness of the $\phi$ function was made in two steps. Firstly, visual comparison between the surface of the specimen at the end of the test (Fig. 9) and the predicted $\phi$ distribution (Fig. 6) gave strength to the hypothesis that $\phi$ can be related to the penetration rate, and, consequently, to the erosion depth at a certain time instant.

Secondly, attention was focused on the erosion rate. As already noticed, in the numerical simulations this parameter is expected to be related to $\Phi$, i.e. the integral of $\phi$. In the experiments, the erosion rate can be estimated from the temporal evolution of the relative mass decrement, shown in Fig. 10.

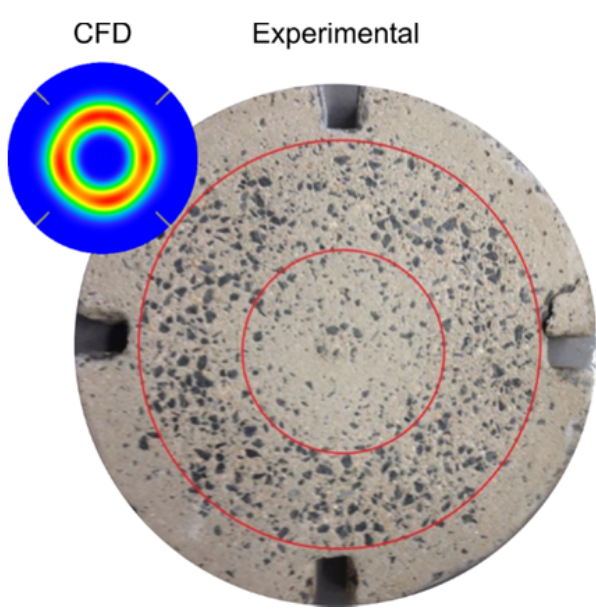

Fig. 9. Surface of the specimen after 24 hours for the low clearance case $(c / T=0.16)$. The image also shows the corresponding $\phi$ distribution, already depicted in Fig. 6.

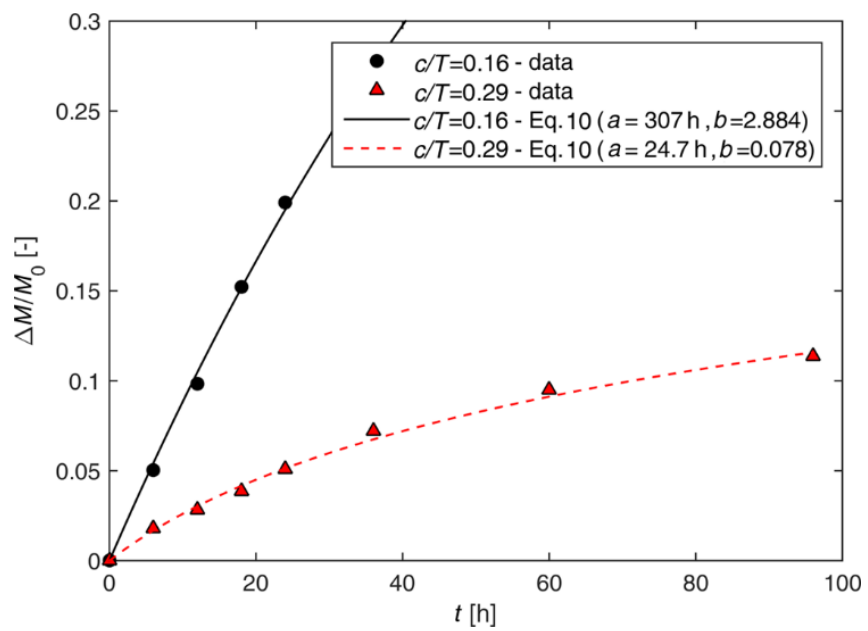

Fig. 10. Time history of the relative mass decrement: experimental data and fitting lines.

Following Horszczaruk (2005), the course of erosion is represented by the following expression:

$$
\frac{\Delta M}{M_{0}}=1-\left(1+\frac{t}{a}\right)^{-b}
$$

The numerical coefficients, $a$ and $b$, have been determined for both tests using interpolation methods, and the corresponding curves are shown in Fig. 10 as well. The evidence indicates that erosion occurs faster for the low clearance case, but the erosion rate, i.e. the derivative of the curves, decreases with time. This behavior is well known in the literature (Horszczaruk, 2004), and it may be caused by different factors, including the inhomogeneous structure of the concrete, the changes in the surface of the specimen due to erosion. The size and the shape degradation may also come into play (Cooke, 1996; Clark, 2002), as this effect may not be fully eliminated by renewing the slurry in the tank every 6 hours.

Since the steady-state Eulerian-Eulerian model employed is not capable in handling such dynamic effects, the comparison against the simulations is made between $\Phi$ and the experimentally estimated erosion rate at $t=0$, when the surface of the specimen is still flat and homogeneous. This quantity, referred 
to as $\dot{E}_{\mathrm{r} 0 \text { exp }}$, is equal to:

$$
\begin{aligned}
\dot{E}_{r 0 \text { exp }}=\left.\frac{d(\Delta M)}{d t}\right|_{t=0} & =\left.\frac{d\left\{M_{0}\left[1-(1+t / a)^{-b}\right]\right\}}{d t}\right|_{t=0} \\
& =\left.M_{0} b\left(1+\frac{t}{a}\right)^{-b-1} \frac{1}{a}\right|_{t=0}=M_{0} \frac{b}{a}
\end{aligned}
$$

The results, summarized in Table 2 , indicate that the rate of variation of $\Phi$ with $c / T$ deviates about $6.5 \%$ from that of $\dot{E}_{\mathrm{r} 0 \text {,exp, }}$ thereby confirming the suitability of $\phi$ for attaining rough estimation of the effect of the fluid dynamic parameters on the erosion of the specimens.

Table 2. Values of $\Phi$, obtained by CFD, and $\dot{E}_{\mathrm{r} 0 \text {,exp }}$, inferred from

\begin{tabular}{|c|c|c|}
\hline$c / T[-]$ & $\begin{array}{c}\text { CFD } \\
\Phi\left[\mathrm{kg} \cdot \mathrm{m}^{2} / \mathrm{s}^{3}\right]\end{array}$ & $\begin{array}{c}\text { Experimental } \\
\dot{E}_{\mathrm{r} 0, \exp }[\mathrm{g} / \mathrm{h}]\end{array}$ \\
\hline 0.16 & 2.72 & 94.0 \\
\hline 0.29 & 0.91 & 33.5 \\
\hline Ratio & 2.99 & 2.81 \\
\hline
\end{tabular}
experiments.

\section{CONCLUSIONS}

A new strategy has been proposed for testing the abrasive erosion of concrete, which involves a strong synergy between numerical simulations and laboratory experiments. As part of this method, a new apparatus has been designed. The equipment is a modified version of the ASTM "underwater method", the differences being the insertion of four lateral baffles in the tank, the change of the impeller with a Pitched Blade one, and the use of sand particles instead of steel balls as abradant (Fig. 2 ). These changes allow activating erosion mechanisms which are closer to those encountered in concrete hydraulic structures, without the need of resorting to complex and expensive setups.

Computational Fluid Dynamics has been employed for the design of the setup. Particularly, the water-sand flow has been simulated in a steady-state by an Euler-Euler model previously developed by two of the authors, and the effect of the rotation of the impeller has been modelled by imposing proper values of mean velocity and turbulence parameters. The effectiveness of the designed equipment was inferred from inspection of the mean velocity field of the solid phase (Fig. 4).

Afterwards, a parameter has been identified to estimate the effect of the fluid dynamics of the system on the erosion of the concrete specimen. A sensitivity analysis allowed establishing the influence on the position of the impeller and its rotational speed on the shape of the erosion scar and the rapidity at which mass removal occurs (Fig. 7 and Table 1). Finally, the succesful comparison against experimental data performed in a prototype apparatus (Figs. 9 and 10) gave strength to the possibility of referring to the proposed parameter for customizing the setup in function of the peculiarities of the experiments.

The next step of this research will consist in the development of specifically-calibrated erosion models for the materials of interests. This will open the way to reliable simulation of concrete hydraulic structures by means of CFD.

Acknowledgements. The authors would like to acknowledge Professor Elzbieta Horszczaruk for kindly providing one of her papers.

\section{REFERENCES}

ASTM C1138M-12, 2012. Standard Test Method for Abrasion Resistance of Concrete (Underwater Method). ASTM International, West Conshohocken, PA.

Cheng, N.S., Law, A.W.K., 2003. Exponential formula for computing effective viscosity. Powder Technol., 129, 156-160.

Clark, H. McI, 2002. Particle velocity and size effects in laboratory slurry erosion measurements OR... do you know what your particles are doing? Tribol. Int., 35, 617-624.

Cooke, R., 1996. Pipeline material evaluation for the Mina Grande hydrohoist system. In: Proc. 13th Int. Conf. on Hydrotransport, Johannesburg, South Africa, pp. 455-477.

Dalfré Filho, J.G., Genovez, A.I.B., Paulon, W.A., 2000. Wear in hydraulic structures and concrete resistance to water-solid mixture. Ingeniería Estructural, 8, 20, 18-22. (In Portuguese.)

Dandapat, R., Deb, A., 2016. A probability based model for the erosive wear of concrete by sediment bearing water. Wear, 350-351, 165-181.

Fokema, M.D., Kresta, S.M., Wood, P.E., 1994. Importance of using the correct impeller boundary conditions for CFD simulations of stirred tanks. Can. J. Chem. Eng., 72, 177-183.

Gnanavelu, A., Kapur, N., Neville, A., Flores, J.F., Ghorbani, N., 2011. A numerical investigation of a geometry independent integrated method to predict erosion rates in slurry erosion. Wear, 271, 712-719.

Graham, J.R., 1998. Erosion of concrete in hydraulic structures. Reported by ACI Committee 210, ACI manual practice, Part 1.

Horszczaruk, E.K., 2000. Abrasion-erosion of concrete. Arch. Civ. Eng., 46, 585-609.

Horszczaruk, E.K., 2004. The model of abrasive wear of concrete in hydraulic structures. Wear, 256, 787-796.

Horszczaruk, E.K., 2005. Abrasion resistance of high-strength concrete in hydraulic structures. Wear, 259, 62-69.

Horszczaruk, E.K, 2008. Mathematical model of abrasive wear of high performance concrete. Wear, 264, 113-118.

Horszczaruk, E.K., 2009. Hydro-abrasive erosion of high performance fiber-reinforced concrete. Wear, 267, 1-4, 110-115.

Jirout, T., Rieger, F., 2011. Impeller design for mixing of suspensions. Chem. Eng. Res. Des., 89, 1144-1151.

Kumar, R.G.B., Sharma, U.K., 2014. Abrasion resistance of concrete containing marginal aggregates. Construction and Building Materials, 66, 712-722.

Launder, B.E., Spalding, D.B., 1972. Mathematical Models of Turbulence. Academic Press, London.

Launder, B.E., Spalding, D.B., 1974. The numerical computation of turbulent flows. Comput. Meth. Appl. Mech. Eng., 3, 269-289.

Liu, Y.W, Yen, T., Hsu, T.H., 2006. Abrasion erosion of concrete by water-borne sand. Cem. Concr. Res., 36, 1814-1820.

Liu, Y.W, Cho, S.W., Hsu, T.H., 2012. Impact abrasion of hydraulic structures concrete. J. Mar. Sci. Tech.-Japan, 20, 3, 253-258.

Mak, A.T.C., 1992. Solid-liquid mixing in mechanically-agitated vessels. PhD Thesis. University College, London UK.

Mansouri, A., Arabnejad, H., Shirazi, S.A., McLaury, B.S., 2015. A combined CFD/experimental methodology for erosion prediction. Wear, 332-333, 1090-1097.

Messa, G.V., Malavasi, S., 2014a. Computational investigation of liquid-solid slurry flow through an expansion in a rectangular duct. J. Hydrol. Hydromech., 62, 234-240.

Messa, G.V., Malavasi, S., 2014b. Numerical prediction of particle distribution of solid-liquid slurries in straight pipies and bends. Eng. Appl. Comput. Fluid Mech., 8, 3, 356-372.

Messa, G.V., Malavasi, S., 2015. Improvements in the numerical prediction of fully-suspended slurry flow in horizontal pipes. Powder Technol., 270, 358-367.

Messa, G.V., Malavasi, S., 2017. The effect of sub-models and parameterizations in the simulation of abrasive jet impingement tests. Wear, 370-371, 59-72.

Messa, G.V., Malin, M., Malavasi S., 2013. Numerical prediction of pressure gradient of slurry flows in horizontal pipes. In: Proc. ASME Pressure Vessels and Piping PVP2013 Conf. (Paris, France). Paper No. PVP2013-97460. 
Messa, G.V., Malin, M., Malavasi S., 2014. Numerical prediction of fully-suspended slurry flow in horizontal pipes. Powder Technol., 256, 61-70.

Mohebi, R., Behfarnia, K., Shojaei, M., 2015. Abrasion resistance of alkali-activated slag concrete designed by Taguchi method. Constr. Build. Mater., 98, 792-798.

NBR 5738, 2003. Moulding and cure of concrete cylindrical and prismatic test pieces. ABNT-Brazilian Association for Technical Standards, Rio de Janeiro, Brazil.

Parsi, M., Najmi, K., Najafifard, F., Hassani, S., McLaury, B.S., Shirazi, S.A., 2014. A comprehensive review of solid particle erosion modeling for oil and gas wells and pipelines applications. J. Nat. Gas Sci. Eng., 21, 850-873.

Picardi, R., Zhao, L., Battaglia, F., 2016. On the ideal grid resolution for two-dimensional eulerian modeling of gas-liquid flows. ASME J. Fluids Eng., 138, No. 114503.

Shiller, L., Naumann, A., 1935. A drag coefficient correlation. Z. Ver. Dtsch. Ing., 77, 318-320.

Spalding, D.B., 1972. A novel finite-difference formulation for differential expresions involving both first and second derivatives. Int. J. Numer. Methods Eng., 4, 551-559.

Spalding, D.B., 1980. Numerical computation of multi-phase fluid flow and heat transfer. In: Taylor, C., Morgan, K. (Eds.): Recent Advances in Numerical Methods in Fluids. Pineridge Press Limited, Swansea.

Yen, T.Y., Hsu, T.H., Liu, Y.W., Chen, S.H., 2007. Influence of class F fly ash on the abrasion-erosion resistance of high-strength concrete. Constr. Build. Mater., 21, 2007, 458-463.

Zwietering, T.N., 1958. Suspending of solid particles in liquid by agitators. Chem. Eng. Sci., 8, 244-253.

Received 13 March 2017

Accepted 15 August 2017

\section{SUPPLEMENTARY MATERIAL}

\section{Appendix A: impeller model}

As is it a common practice in stirred tank simulations, the effect of the rotating impeller on the mean flow was modelled by fixing the velocities of both phases, the turbulent kinetic energy, and the dissipation rate in a fluid region located in correspondence of the impeller (green coloured in Fig. 3).

These variables are assumed axi-symmetrically distributed. Their radial profiles are obtained by interpolating the data reported by Fokema et al. (1994) for a relative clearance of 0.5. In order to allow extension to different blade diameters, hub diameters, and impeller rotational velocities, the interpolation curves are shown in Fig. 1A in dimensionless form, making reference to the following variables, $U_{\text {imp }} / V_{\text {tip }}, V_{\text {imp }} / V_{\text {tip }}$, $W_{\text {imp }} / V_{\text {tip }}, k_{\text {imp }} / V_{\text {tip }}^{2}, \varepsilon_{\text {imp }} / V_{\text {tip. }}^{3}$. In the above, $U_{\text {imp }}, V_{\text {imp }}, W_{\text {imp }}$ are the induced mean velocity components along the azimuthal, radial, and axial direction, respectively, $k_{\text {imp }}$ and $\varepsilon_{\text {imp }}$ are the induced turbulent kinetic energy and dissipation rate, $R_{\text {imp }}$ is the radius of the impeller, and the tip velocity, $V_{\text {tip }}$, and the dimensionless radial coordinate, $\zeta$, are given by:

$$
\begin{aligned}
& V_{\text {tip }}=2 \pi \Omega R_{\text {imp }} \\
& \zeta=\frac{r-R_{\text {hub }}}{R_{\text {imp }}-R_{\text {hub }}}
\end{aligned}
$$

where $R_{\text {hub }}$ is the radius of the hub. The equations corresponding to the profiles shown in Fig. 1A are reported below:

$$
\begin{aligned}
& \frac{U_{\text {imp }}}{V_{\text {tip }}}=0.313 \cdot \frac{r}{R_{\text {imp }}} \\
& \frac{V_{\text {imp }}}{V_{\text {tip }}}=0.262 \zeta^{3}-0.572 \zeta^{2}+0.349 \zeta
\end{aligned}
$$

$$
\begin{aligned}
& \frac{W_{\text {imp }}}{V_{\text {tip }}}=2.3997 \zeta^{4}-4.0526 \zeta^{3}+2.6984 \zeta^{2}-1.2961 \zeta \\
& \frac{k}{V_{\text {tip }}^{2}}=0.2991 \zeta^{3}-0.3605 \zeta^{2}+0.1564 \zeta \\
& \frac{\varepsilon}{V_{\text {tip }}^{3}}=5.1254 \zeta^{3}-5.547 \zeta^{2}+1.7241 \zeta
\end{aligned}
$$

In order to account for the obstacle produced by the hub, $V_{\text {imp, }}$, $W_{\text {imp }}, k_{\text {imp }}$, and $\varepsilon_{\text {imp }}$ were set to a null value for $\zeta<0\left(r<R_{\text {hub }}\right)$.

The mean velocity profiles reported above have been applied to both the fluid and the solids, thereby assuming absence of slip between the phases in the impeller region.
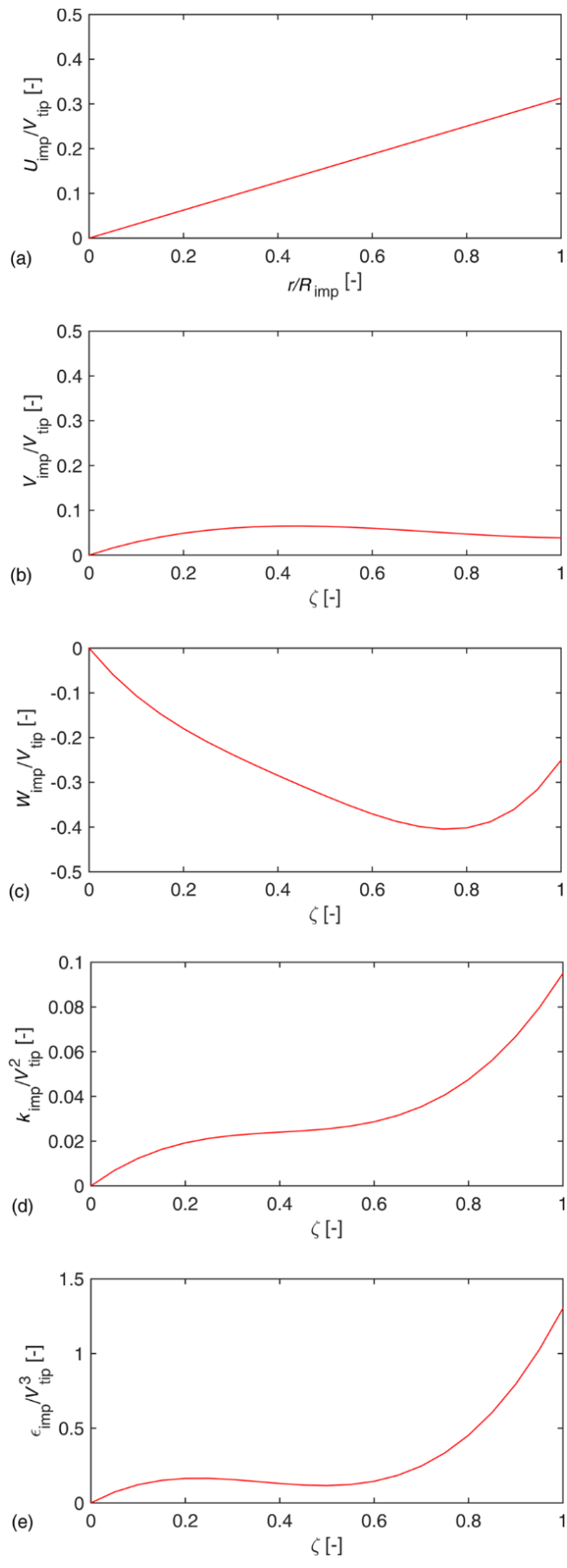

Fig. 1A. Radial profiles of the mean velocity and turbulent parameters imposed in the impeller region. 